\title{
Evaluation of the safety and efficacy of transcatheter aortic valve implantation in patients with a severe stenotic bicuspid aortic valve in a Chinese population*
}

\author{
Xian-bao LIU ${ }^{1}$, Ju-bo JIANG ${ }^{1}$, Qi-jing ZHOU ${ }^{2}$, Zhao-xia $\mathrm{PU}^{3}$, Wei HE${ }^{4}$, Ai-qiang DONG ${ }^{5}$, Yan FENG ${ }^{1}$, \\ Jun JIANG ${ }^{1}$, Yong SUN ${ }^{1}$, Mei-xiang XIANG ${ }^{1}$, Yu-xin $\mathrm{HE}^{1}$, You-qi FAN ${ }^{1}$, Liang DONG ${ }^{1}$, Jian-an WANG ${ }^{\dagger 1}$ \\ $\left({ }^{1}\right.$ Cardiovascular Key Lab of Zhejiang Province, Department of Cardiology, the Second Affiliated Hospital, \\ School of Medicine, Zhejiang University, Hangzhou 310009, China) \\ ( ${ }^{2}$ Department of Radiology, the Second Affiliated Hospital, School of Medicine, Zhejiang University, Hangzhou 310009, China) \\ ( ${ }^{3}$ Department of Echocardiography, the Second Affiliated Hospital, School of Medicine, Zhejiang University, Hangzhou 310009, China) \\ ( ${ }^{4}$ Department of Anesthesiology, the Second Affiliated Hospital, School of Medicine, Zhejiang University, Hangzhou 310009, China) \\ $\left({ }^{5}\right.$ Department of Cardiosurgery, the Second Affiliated Hospital, School of Medicine, Zhejiang University, Hangzhou 310009, China) \\ †E-mail: jian_an_wang@yahoo.com \\ Received Jan. 21, 2015; Revision accepted Feb. 23, 2015; Crosschecked Feb. 26, 2015
}

\begin{abstract}
Objective: The purpose of this study is to evaluate the safety and efficacy of transcatheter aortic valve implantation (TAVI) in patients with a severe stenotic bicuspid aortic valve (BAV) in a Chinese population. While several groups have reported the feasibility, efficacy, and safety of TAVI for patients with a BAV, worldwide experience of the technique is still limited, especially in China. Methods: From March 2013 to November 2014, high surgical risk or inoperable patients with symptomatic severe aortic stenosis (AS) who had undergone TAVI at our institution were selected for inclusion in our study. Results were compared between a BAV group and a tricuspid aortic valve (TAV) group. Results: Forty patients were included in this study, $15(37.5 \%)$ of whom were identified as having a BAV. In the BAV group, the aortic valve area was smaller $\left((0.47 \pm 0.13)\right.$ vs. $\left.(0.59 \pm 0.14) \mathrm{cm}^{2}\right)$, the ascending aortic diameter was larger $((40.4 \pm 4.4)$ vs. $(36.4 \pm 4.3) \mathrm{mm})$, and the concomitant aortic regurgitation was lower. No significant differences were found between the groups in the other baseline characteristics. No differences were observed either in the choice of access or valve size. The procedural success achieved in this study was $100 \%$. There were no differences between groups in device success $(86.7 \%$ vs. $88.0 \%)$, 30 -d mortality $(6.7 \%$ vs. $8.0 \%)$, or 30 -d combined end point $(13.3 \%$ vs. $12.0 \%$ ). The incidences of new pacemaker implantation, paravalvular regurgitation and other complications, recovery of left ventricle ejection fraction and heart function were similar in both groups. Conclusions: Patients with a severely stenotic BAV can be treated with TAVI, and their condition after treatment should be similar to that of people with a TAV.
\end{abstract}

Key words: Aortic valve stenosis, Bicuspid aortic valve, Transcatheter aortic valve implantation doi: $10.1631 /$ jzus.B1500017

\section{Introduction}

\footnotetext{
Corresponding author

* Project supported by the Fundamental Research Funds from Zhejiang University (No. 2012FZA7008), China

(D) ORCID: Xian-bao LIU, http://orcid.org/0000-0002-2891-7019; Jian-an WANG, http://orcid.org/0000-0003-0409-8941

(c) Zhejiang University and Springer-Verlag Berlin Heidelberg 2015
}

Transcatheter aortic valve implantation (TAVI) has emerged as a promising treatment strategy for patients with severe symptomatic aortic stenosis (AS), whose condition has been carefully evaluated as being high surgical risk or inoperable patients (Leon et al., 
2010; Smith et al., 2011; Adams et al., 2014). Despite the great improvements achieved in recent years, unfortunately, TAVI for patients with a bicuspid aortic valve (BAV) is still deemed to be a relative contraindication because of the exclusion of these patients from most clinical trials (Leon et al., 2010; Smith et al., 2011). One possible concern is the unfavorable anatomy of a BAV, which will result in elliptical deployment of a bioprosthesis, increase the procedural complications including residual aortic regurgitation, coronary obstruction, annulus rupture and aortic dissection, and decrease the durability of the bioprosthesis (Zegdi et al., 2008; 2010; Wijesinghe et al., 2010; Himbert et al., 2012). However, $\mathrm{BAV}$ is the most common congenital heart disease, occurring in $0.5 \%$ to $2.0 \%$ of the general population (Roberts, 1970; Nistri et al., 2005; Tutar et al., 2005), and has been observed in about $50 \%$ of aortic valves resected during surgical aortic valve replacement (SAVR) (Roberts and Ko, 2005). Although several groups have reported the feasibility, efficacy, and safety of TAVI for patients with a BAV (Wijesinghe et al., 2010; Himbert et al., 2012; Hayashida et al., 2013; Bauer et al., 2014; Costopoulos et al., 2014; Kochman et al., 2014), global experience of the procedure is lacking, especially in China.

The purpose of this study is to evaluate the safety and efficacy of TAVI in patients with a severe stenotic BAV in a Chinese population.

\section{Subjects and methods}

From March 2013, consecutive patients with symptomatic severe AS (valve area $<1.0 \mathrm{~cm}^{2}$ ), who had undergone TAVI at our institution, were prospectively included in this study. The inclusion criteria for candidates with TAVI included surgical high risk defined as logistic EuroSCORE $\geq 20 \%$, or other risk factors not included in the risk scores (e.g. frailty, porcelain aorta, previous chest radiotherapy, and advanced age). The decision to proceed with TAVI was reviewed by a dedicated heart valve team, including experienced interventional cardiologists, general cardiologists, cardiac surgeons, anesthesiologists, and cardiac radiologists. Between March 2013 and November 2014, a total of 40 patients underwent TAVI, $15(37.5 \%)$ of whom had a BAV.
According to a previous report (Hayashida et al., 2013), BAVs are defined as having deformed aortic valves with two functional cusps forming a valve mechanism with less than three zones of parallel apposition between the cusps. BAVs are classified into three types depending on the number of raphes: type 0 , valves with no raphes; type 1, valves with one raphe; and type 2, valves with two raphes (Sievers and Schmidtke, 2007). Patients with functional BAVs due to commissural fusion in tricuspid aortic valves (TAVs) were not included in the BAV group. The diagnosis of BAV was made by imaging specialists through transthoracic echocardiography (TTE) and dualsource computed tomography (DSCT) in all cases.

As to procedure aspects, the selection of the transfemoral access or alternative approaches, including the subclavian artery and aorta, depended on the size, calcification, and tortuosity of the iliofemoral arterial access performed by a DSCT. The choices of general or local anesthesia, cutting down or puncture of vascular access were made according to each patient's condition and the operator's experience. Two valve prostheses, CoreValve (Medtronic Inc., Minneapolis, Minnesota, USA) in diameters of 23, 26, 29, and $31 \mathrm{~mm}$ and VENUS A (Venus Medtech, Hangzhou, China) in diameters of 23, 26, 29, and $32 \mathrm{~mm}$, were used in this study. All patients received dual antiplatelet therapy daily before TAVI or were administered a loading dosage of $300 \mathrm{mg}$ of clopidogrel and $300 \mathrm{mg}$ of aspirin before the procedure. After the successful preparation of the vascular access, a bolus of intravenous heparin $(70 \mathrm{IU} / \mathrm{kg})$ was injected to achieve an activated clotting time of 250 to $300 \mathrm{~s}$, which was checked and adjusted every $30 \mathrm{~min}$ thereafter. Clopidogrel at $75 \mathrm{mg} / \mathrm{d}$ was used for 3 to 6 months after the procedure and aspirin at $100 \mathrm{mg} / \mathrm{d}$ will be continued for life if there were no contraindications. Oral anticoagulation was administered if necessary. Follow-up data from all the patients were collected at $30 \mathrm{~d}$, including clinical assessment, electrocardiography, and TTE data.

Clinical end points and device success were defined according to the standardized definitions for TAVI published by the Valve Academic Research Consortium (Leon et al., 2011). The main end points derived from this study were the all-cause $30-\mathrm{d}$ mortality, a 30-d safety composite end point, and device success. The combined $30-\mathrm{d}$ safety end points included 
all-cause mortality, major stroke, life-threatening bleeding, acute kidney injury stage 3 (including renal replacement therapy), periprocedural myocardial infarction, major vascular complications and repeated procedure for valve-related dysfunction (surgical or interventional therapy). Device success was defined as successful vascular access, delivery and deployment of a single prosthesis in the proper anatomical location, successful retrieval of the delivery system and intended performance of the prosthetic heart valve (aortic valve area $>1.2 \mathrm{~cm}^{2}$ and mean aortic valve gradient $<20 \mathrm{mmHg}$ or peak velocity $<3 \mathrm{~m} / \mathrm{s}$, without moderate or severe prosthetic valve aortic regurgitation) (Leon et al., 2011).

Qualitative variables are expressed as numbers or percentages and quantitative variables as mean \pm standard deviation (SD). The unpaired Student's $t$-test or Wilcoxon rank-sum test was used to compare quantitative variables according to the variable's distribution. Qualitative variables were compared using the Fisher exact test. Statistical significance was defined as $P<0.05$. The data were analyzed using SPSS statistics 21.0 (SPSS Inc., Chicago, Illinois, USA).

Table 1 Baseline characteristics of patients in this study

\begin{tabular}{|c|c|c|c|c|}
\hline Characteristics & $\begin{array}{c}\text { Total } \\
(n=40)\end{array}$ & $\begin{array}{c}\text { BAV } \\
(n=15)\end{array}$ & $\begin{array}{c}\text { TAV } \\
(n=25)\end{array}$ & $P$-value \\
\hline Age (year) & $75.7 \pm 5.5$ & $75.4 \pm 5.7$ & $75.8 \pm 5.5$ & 0.81 \\
\hline Men & $26(65.0 \%)$ & $9(60.0 \%)$ & $17(68.0 \%)$ & 0.61 \\
\hline BMI $\left(\mathrm{kg} / \mathrm{m}^{2}\right)$ & $24.4 \pm 3.4$ & $23.6 \pm 4.8$ & $21.7 \pm 3.1$ & 0.32 \\
\hline Hypertension & $19(47.5 \%)$ & $5(33.3 \%)$ & $14(56.0 \%)$ & 0.17 \\
\hline Diabetes mellitus & $3(7.5 \%)$ & $0(0 \%)$ & $3(12.0 \%)$ & 0.44 \\
\hline Coronary artery disease & $12(30.0 \%)$ & $3(20.0 \%)$ & $9(36.0 \%)$ & 0.48 \\
\hline Previous MI & $0(0 \%)$ & $0(0 \%)$ & $0(0 \%)$ & 1.00 \\
\hline Previous CABG & $0(0 \%)$ & $0(0 \%)$ & $0(0 \%)$ & 1.00 \\
\hline Previous PCI & $6(15.0 \%)$ & $3(20.0 \%)$ & $3(12.0 \%)$ & 0.82 \\
\hline Previous valve surgery & $0(0 \%)$ & $0(0 \%)$ & $0(0 \%)$ & 1.00 \\
\hline Previous pacemaker/ICD implantation & $0(0 \%)$ & $0(0 \%)$ & $0(0 \%)$ & 1.00 \\
\hline Cerebral vascular disease & $2(5.0 \%)$ & $0(0 \%)$ & $2(8.0 \%)$ & 0.52 \\
\hline Peripheral vascular disease & $6(15.0 \%)$ & $2(13.3 \%)$ & $4(16.0 \%)$ & 1.00 \\
\hline Atrial fibrillation & $3(7.5 \%)$ & $1(6.7 \%)$ & $2(8.0 \%)$ & 1.00 \\
\hline COPD & $8(20.0 \%)$ & $4(26.7 \%)$ & $4(16.0 \%)$ & 0.68 \\
\hline Chronic renal insufficiency (eGFR <60 ml/min) & $16(40.0 \%)$ & $4(26.7 \%)$ & $12(48.0 \%)$ & 0.18 \\
\hline Pulmonary hypertension (SPAP $>60$ mmHg) & $5(12.5 \%)$ & $2(13.3 \%)$ & $3(12.0 \%)$ & 1.00 \\
\hline Malignancy & $4(10.0 \%)$ & $0(0 \%)$ & $4(16.0 \%)$ & 0.28 \\
\hline NYHA class III-IV & $34(85.0 \%)$ & $13(86.7 \%)$ & $21(84.0 \%)$ & 1.00 \\
\hline Logistic EuroSCORE (\%) & $19.6 \pm 13.6$ & $16.1 \pm 11.1$ & $21.8 \pm 14.7$ & 0.22 \\
\hline STS score $(\%)$ & $7.1 \pm 5.2$ & $5.6 \pm 4.1$ & $7.5 \pm 5.9$ & 0.18 \\
\hline LVEF (\%) & $51.0 \pm 12.5$ & $51.1 \pm 12.6$ & $51.0 \pm 12.7$ & 0.90 \\
\hline $\mathrm{LVEF}<40 \%$ & $9(22.5 \%)$ & $3(20.0 \%)$ & $6(24.0 \%)$ & 1.00 \\
\hline Aortic-valve area $\left(\mathrm{cm}^{2}\right)$ & $0.54 \pm 0.15$ & $0.47 \pm 0.13$ & $0.59 \pm 0.14$ & 0.02 \\
\hline Aortic-valve gradient $(\mathrm{mmHg})$ & $58.0 \pm 16.8$ & $64.1 \pm 19.5$ & $54.3 \pm 14.2$ & 0.08 \\
\hline Peak velocity $(\mathrm{m} / \mathrm{s})$ & $4.9 \pm 0.7$ & $5.1 \pm 0.8$ & $4.9 \pm 0.6$ & 0.29 \\
\hline $\mathrm{AR}(0-4)$ & $2.2 \pm 0.8$ & $1.8 \pm 0.9$ & $2.4 \pm 0.7$ & 0.01 \\
\hline $\mathrm{AR}>2$ & $15(37.5 \%)$ & $2(13.3 \%)$ & $13(52.0 \%)$ & 0.01 \\
\hline Mitral regurgitation $(0-4)$ & $2.3 \pm 0.6$ & $2.3 \pm 0.7$ & $2.2 \pm 0.6$ & 0.32 \\
\hline Mean annulus diameter, CT-measured (mm) & $24.6 \pm 2.4$ & $24.7 \pm 1.8$ & $24.6 \pm 2.7$ & 0.96 \\
\hline Short-axis annulus size, CT-measured (mm) & $21.5 \pm 2.3$ & $21.8 \pm 1.9$ & $21.3 \pm 2.5$ & 0.58 \\
\hline Long-axis annulus size, CT-measured (mm) & $27.6 \pm 3.1$ & $27.7 \pm 3.1$ & $27.5 \pm 3.2$ & 0.87 \\
\hline Ascending aorta diameter, CT-measured (mm) & $37.9 \pm 4.7$ & $40.4 \pm 4.4$ & $36.4 \pm 4.3$ & 0.01 \\
\hline
\end{tabular}

Data are expressed as mean $\pm \mathrm{SD}$ or number (percentage), except $P$-values. BAV: bicuspid aortic valve; TAV: tricuspid aortic valve; BMI: body mass index; MI: myocardial infarction; CABG: coronary artery bypass graft; PCI: percutaneous coronary intervention; ICD: implantable cardioverter defibrillator; COPD: chronic obstructive pulmonary disease; eGFR: estimated glomerular filtration rate; SPAP: systolic pulmonary artery pressure; NYHA: New York Heart Association; STS: Society of Thoracic Surgeons; LVEF: left ventricular ejective fraction; $A R$ : aortic regurgitation 


\section{Results}

From March 2013 to November 2014, a total of 40 patients with symptomatic severe AS undergoing TAVI were consecutively included in this study. Fifteen of these patients $(37.5 \%)$ were identified as having a BAV by DSCT and TTE. The BAV group consisted of type 0 (11 cases) and type 1 ( 4 cases) according to previously described criteria (Sievers and Schmidtke, 2007). The aortic valve area was much smaller $((0.47 \pm$ $0.13)$ vs. $\left.(0.59 \pm 0.14) \mathrm{cm}^{2}, P=0.02\right)$ and the mean gradient was higher $((64.1 \pm 19.5)$ vs. $(54.3 \pm 14.2) \mathrm{mmHg}$, $P=0.08)$ in the BAV group, and concomitant aortic regurgitation occurred more often in the TAV group as shown by TTE (Table 1). The ascending aortic diameter was larger $((40.4 \pm 4.4)$ vs. $(36.4 \pm 4.3) \mathrm{mm}$, $P=0.01)$ in the BAV group according to DSCT evaluation. No significant differences were found between the BAV and TAV groups in other baseline and clinical characteristics (Table 1).

CoreValve was implanted in $72.5 \%$ of cases and VENUS A in the rest. The transfemoral approach was used in $90 \%$ of cases, a subclavian approach in only one patient and direct aortic access in three patients. A 29-mm prosthesis was most frequently applied, whether CoreValve or VENUS A. However, no difference was observed between the BAV and TAV groups in either the choice of access or the valve size (Table 2).

A procedural success rate of $100 \%$ was achieved in this study. Device success was $86.8 \%$ in the BAV group and $88.0 \%$ in the TAV group $(P=1.00)$, and the incidence of second valve implantation was similar in each group. New pacemakers were implanted in 5 patients (12.5\%), 2 from the BAV group (13.3\%) and 3 from the TAV group (12.0\%). No annulus rupture, coronary occlusion or conversion to open heart surgery occurred. There were no significant differences between the two groups in bleeding, cerebrovascular accidents, major vascular complications, or myocardial infarction after the procedure. No acute kidney injury or new onset atrial fibrillation was observed in either group (Table 3).

All patients were followed up for one month. There were no differences between the two groups in the incidence of $30-\mathrm{d}$ mortality $(6.7 \%$ vs. $8.0 \%$, $P=1.00)$ or $30-\mathrm{d}$ combined safety end point $(12.5 \%$ vs. $12.0 \%, P=1.00$ ) (Table 3). Clinical follow-up showed that the heart function status in both groups had obviously improved, and heart function with NYHA III was found in only one patient with TAV anatomy. Echocardiography showed no differences in aortic

Table 2 Delivery access, type, and size of implanted valves

\begin{tabular}{lcccc}
\hline \multirow{2}{*}{ Variable } & \multicolumn{3}{c}{ Case number } & \multirow{2}{*}{$P$-value } \\
\cline { 2 - 3 } & Total $(n=40)$ & BAV $(n=15)$ & TAV $(n=25)$ & 0.78 \\
\hline Access & $29(72.5 \%)$ & $10(66.7 \%)$ & $19(76.0 \%)$ & 1.00 \\
CoreValve & $27(67.5 \%)$ & $10(66.7 \%)$ & $17(68.0 \%)$ & 1.00 \\
Transfemoral & $1(2.5 \%)$ & $0(0 \%)$ & $1(4.0 \%)$ & 1.00 \\
Transubclavian & $1(2.5 \%)$ & $0(0 \%)$ & $1(4.0 \%)$ & 0.78 \\
Transaortic & $11(27.5 \%)$ & $5(33.3 \%)$ & $6(24.0 \%)$ & 1.00 \\
VANUS A & $9(22.5 \%)$ & $3(20.0 \%)$ & $6(24.0 \%)$ & 1.00 \\
Transfemoral & $0(0 \%)$ & $0(0 \%)$ & $0(0 \%)$ & 0.14 \\
Transubclavian & $2(5.0 \%)$ & $2(13.3 \%)$ & $0(0 \%)$ & 1.00 \\
Transaortic & $36(90.0 \%)$ & $13(86.7 \%)$ & $23(92.0 \%)$ & 1.00 \\
Transfemoral access TOTAL & & & & 0.71 \\
Valve size & $1(2.5 \%)$ & $0(0 \%)$ & $1(4.0 \%)$ & 0.22 \\
CoreValve & $5(12.5 \%)$ & $1(6.7 \%)$ & $4(16.0 \%)$ & 0.28 \\
$23 \mathrm{~mm}$ & $19(47.5 \%)$ & $9(60.0 \%)$ & $10(40.0 \%)$ & 1.00 \\
$26 \mathrm{~mm}$ & $4(10.0 \%)$ & $0(0 \%)$ & $4(16.0 \%)$ & 0.64 \\
$29 \mathrm{~mm}$ & $1(2.5 \%)$ & $0(0 \%)$ & $1(4.0 \%)$ & 0.82 \\
$31 \mathrm{~mm}$ & $3(7.5 \%)$ & $2(13.3 \%)$ & $1(4.0 \%)$ & $3(12.0 \%)$ \\
VANUS A & $6(15.0 \%)$ & $3(20.0 \%)$ & $1(4.0 \%)$ & \\
$23 \mathrm{~mm}$ & $1(2.5 \%)$ & $0(0 \%)$ & & \\
$26 \mathrm{~mm}$ & & & &
\end{tabular}

${ }^{*}$ Data are expressed as number (percentage). BAV: bicuspid aortic valve; TAV: tricuspid aortic valve 
valve area, mean gradient, peak velocity, or paravalvular regurgitation. No significant difference was observed in the amelioration of the left ventricular ejection fraction (LVEF), which increased from $(51.1 \pm 12.6) \%$ to $(58.3 \pm 7.6) \%$ in the BAV group and from $(51.0 \pm 12.7) \%$ to $(57.4 \pm 6.9) \%$ in the TAV group (Table 3).

\section{Discussion}

In this study, the characteristics and clinical outcomes of patients with severe symptomatic BAV who underwent TAVI, were compared to those with a TAV. We found that the incidences of 30-d mortality, 30-d combined safety end point, and device success

Table 3 Intraprocedural, postprocedural, and clinical outcomes

\begin{tabular}{|c|c|c|c|c|}
\hline Variable & $\begin{array}{c}\text { Total } \\
(n=40)\end{array}$ & $\begin{array}{c}\text { BAV } \\
(n=15)\end{array}$ & $\begin{array}{c}\text { TAV } \\
(n=25)\end{array}$ & $P$-value \\
\hline \multicolumn{5}{|l|}{ Intraprocedural } \\
\hline Annulus rupture & $0(0 \%)$ & $0(0 \%)$ & $0(0 \%)$ & 1.00 \\
\hline Coronary occlusion & $0(0 \%)$ & $0(0 \%)$ & $0(0 \%)$ & 1.00 \\
\hline Conversion to open heart surgery & $0(0 \%)$ & $0(0 \%)$ & $0(0 \%)$ & 1.00 \\
\hline Valve-in-valve & $5(12.5 \%)$ & $2(13.3 \%)$ & $3(12.0 \%)$ & 1.00 \\
\hline Procedural success & $40(100 \%)$ & $15(100 \%)$ & $25(100 \%)$ & 1.00 \\
\hline Device success & $35(87.5 \%)$ & $13(86.7 \%)$ & $22(88.0 \%)$ & 1.00 \\
\hline \multicolumn{5}{|l|}{ Postprocedural } \\
\hline Transfusions $\geq 4 \mathrm{U}$ & $1(2.5 \%)$ & $0(0 \%)$ & $1(4.0 \%)$ & 1.00 \\
\hline Bleeding (life threatening) & $1(2.5 \%)$ & $0(0 \%)$ & $1(4.0 \%)$ & 1.00 \\
\hline Bleeding (major) & $6(15.0 \%)$ & $1(6.7 \%)$ & $5(20.0 \%)$ & 0.49 \\
\hline Myocardial infarction & $1(2.5 \%)$ & $1(6.7 \%)$ & $0(0 \%)$ & 0.38 \\
\hline \multicolumn{5}{|l|}{ Cerebrovascular accident } \\
\hline TIA & $0(0 \%)$ & $0(0 \%)$ & $0(0 \%)$ & 1.00 \\
\hline Stroke & $2(5.0 \%)$ & $1(6.7 \%)$ & $1(4.0 \%)$ & 1.00 \\
\hline minor & $2(5.0 \%)$ & $1(6.7 \%)$ & $1(4.0 \%)$ & 1.00 \\
\hline major & $0(0 \%)$ & $0(0 \%)$ & $0(0 \%)$ & 1.00 \\
\hline Major vascular complications & $1(2.5 \%)$ & $1(6.7 \%)$ & $0(0 \%)$ & 0.38 \\
\hline Acute kidney injury & $0(0 \%)$ & $0(0 \%)$ & $0(0 \%)$ & 1.00 \\
\hline New pacemaker & $5(12.5 \%)$ & $2(13.3 \%)$ & $3(12.0 \%)$ & 1.00 \\
\hline New onset atrial fibrillation & $0(0 \%)$ & $0(0 \%)$ & $0(0 \%)$ & 1.00 \\
\hline Valve-related dysfunction requiring repeat procedure & $0(0 \%)$ & $0(0 \%)$ & $0(0 \%)$ & 1.00 \\
\hline Aortic-valve area $\left(\mathrm{cm}^{2}\right)$ & $1.58 \pm 0.30$ & $1.55 \pm 0.15$ & $1.61 \pm 0.35$ & 0.49 \\
\hline Aortic-valve gradient (mmHg) & $10.5 \pm 3.8$ & $9.6 \pm 3.1$ & $11.0 \pm 4.2$ & 0.38 \\
\hline Peak velocity (m/s) & $2.2 \pm 0.4$ & $2.2 \pm 0.3$ & $2.3 \pm 0.4$ & 0.36 \\
\hline LVEF (\%) & $57.7 \pm 7.1$ & $58.3 \pm 7.6$ & $57.4 \pm 6.9$ & 0.72 \\
\hline $\operatorname{PVLR}(0-4)$ & $1.6 \pm 0.7$ & $1.8 \pm 0.4$ & $1.6 \pm 0.8$ & 0.43 \\
\hline PVLR $>2$ & $1(2.5 \%)$ & $0(0 \%)$ & $1(4.0 \%)$ & 1.00 \\
\hline $\operatorname{MR}(0-4)$ & $1.9 \pm 0.7$ & $2.1 \pm 0.5$ & $1.9 \pm 0.8$ & 0.23 \\
\hline $\mathrm{MR}>2$ & $5(12.5 \%)$ & $2(13.3 \%)$ & $3(12.0 \%)$ & 1.00 \\
\hline NYHA III-IV & $1(2.5 \%)$ & $0(0 \%)$ & $1(4.0 \%)$ & 1.00 \\
\hline \multicolumn{5}{|l|}{ 30-d mortality } \\
\hline From any causes & $3(7.5 \%)$ & $1(6.7 \%)$ & $2(8.0 \%)$ & 1.00 \\
\hline From cardiac causes & $1(2.5 \%)$ & $0(0 \%)$ & $1(4.0 \%)$ & 1.00 \\
\hline 30-d composite safety end point & $5(12.5 \%)$ & $2(13.3 \%)$ & $3(12.0 \%)$ & 1.00 \\
\hline
\end{tabular}

Data are expressed as mean $\pm \mathrm{SD}$ or number (percentage), except $P$-values. BAV: bicuspid aortic valve; TAV: tricuspid aortic valve; TIA: transient ischemic attack; LVEF: left ventricular ejective fraction; PVLR: paravalvular regurgitation; MR: mitral regurgitation; NYHA: New York Heart Association 
of the BAV group were similar to those of the TAV group. The results of this study suggest that TAVI in high surgical risk or inoperable patients with severely stenotic BAV is safe and feasible.

Many studies and some case reports have demonstrated a positive effect of TAVI when used on BAV patients whose condition is inoperable or has a high surgical risk (Wijesinghe et al., 2010; Himbert et al., 2012; Hayashida et al., 2013; Bauer et al., 2014; Costopoulos et al., 2014; Kochman et al., 2014). However, experience from Asian populations, especially from China, is scarce. To our knowledge, this is the first report to compare TAVI in severe AS patients with a BAV versus those with a TAV in a Chinese population. In view of the good performance of the TAVI procedure in patients with a BAV, our results indicate that TAVI can be safely and effectively applied to BAV patients in Chinese populations.

Similar to previous studies of the use of TAVI on patients with a BAV, no increase in complications, such as residual aortic regurgitation, coronary obstruction, annulus rupture, or aortic dissection, was observed in our study. To achieve satisfactory results from the procedure, the selection of the access route according to the anatomy and angulation, choice of balloon size for pre-dilation and valve size, consideration of the implanting depth and whether post-dilation should be used, and how to do the post-dilation are very important. However, no ideal software for the TAVI procedure is available to guide the selection of an appropriate valve for individuals, especially for patients with a BAV. Thus, the experience of the heart valve team and careful discussion before the procedure are crucial to avoid complications during the procedure.

As noted, the proportion of patients with a BAV $(15$ of $40,37.5 \%)$ in this study was much higher than those of previous studies in Europe (3.0\% to 9.2\%) (Himbert et al., 2012; Hayashida et al., 2013; Bauer et al., 2014; Costopoulos et al., 2014; Kochman et al., 2014). This phenomenon has also been observed in almost every center that performs TAVI in China, and in some centers as many as $50 \%$ of the patients who underwent TAVI had a BAV. Although functional BAVs due to commissural fusion of TAVs may be included in estimates in some centers, the proportion of true BAVs is actually very high in China. In this study, the percentage of BAVs with type 0 was $73.3 \%$.
However, epidemic data on the prevalence of BAVs show that the percentage of patients with a severely stenotic BAV among all patients with severe AS is very low. Therefore, further epidemic investigations in the field of AS in Chinese populations are urgently needed.

Aortic dilation is frequently associated with BAV, and surgery of the ascending aorta is indicated in cases of ascending aortic diameter $>45 \mathrm{~mm}$ when SAVR is scheduled or ascending aortic diameter $>50 \mathrm{~mm}$ in the presence of other risk factors (Erbel et al., 2014). In our study, patents with ascending aortic diameter $>50 \mathrm{~mm}$ were excluded. Only two patients with ascending aortic diameter $>45 \mathrm{~mm}$ (48.4 and $49.4 \mathrm{~mm}$, respectively) were included. However, the surgical risk evaluation suggested these two patients were at high risk and surgery was not recommended by our heart valve team. Thus, careful follow-up of all the patients with aortic dilation should be applied and surgery will be considered when the aortic diameter increased very quickly in accordance with the suggestions of the heart valve team.

Limitations of this study include the small sample size and the short-term of the follow-up. Furthermore, data from only single center were collected, making it difficult to generalize about the use of TAVI for treating BAV patients. Multi-center registries with large samples and long-term follow-up results will make the conclusion more convincing.

In conclusion, in Chinese populations, patients with a severely stenotic BAV can be treated with TAVI, and their condition after treatment should be similar to that of patients with a TAV.

\section{Compliance with ethics guidelines}

Xian-bao LIU, Ju-bo JIANG, Qi-jing ZHOU, Zhao-xia PU, Wei HE, Ai-qiang DONG, Yan FENG, Jun JIANG, Yong SUN, Mei-xiang XIANG, Yu-xin HE, You-qi FAN, Liang DONG, and Jian-an WANG declare that they have no conflict of interest.

All procedures followed were in accordance with the ethical standards of the responsible committee on human experimentation (institutional and national) and with the Helsinki Declaration of 1975, as revised in 2008 (5). Informed consent was obtained from all patients for being included in the study. Additional informed consent was obtained from all patients for whom identifying information is included in this article.

\section{References}

Adams, D.H., Popma, J.J., Reardon, M.J., 2014. Transcatheter 
aortic-valve replacement with a self-expanding prosthesis N. Engl. J. Med., 371(10):967-968. [doi:10.1056/NEJMc 1408396]

Bauer, T., Linke, A., Sievert, H., et al., 2014. Comparison of the effectiveness of transcatheter aortic valve implantation in patients with stenotic bicuspid versus tricuspid aortic valves (from the German TAVI Registry). Am. $J$. Cardiol., 113(3):518-521. [doi:10.1016/j.amjcard.2013. 10.023]

Costopoulos, C., Latib, A., Maisano, F., et al., 2014. Comparison of results of transcatheter aortic valve implantation in patients with severely stenotic bicuspid versus tricuspid or nonbicuspid valves. Am. J. Cardiol., 113(8): 1390-1393. [doi:10.1016/j.amjcard.2014.01.412]

Erbel, R., Aboyans, V., Boileau, C., et al., 2014. 2014 ESC Guidelines on the diagnosis and treatment of aortic diseases: document covering acute and chronic aortic diseases of the thoracic and abdominal aorta of the adult. The Task Force for the Diagnosis and Treatment of Aortic Diseases of the European Society of Cardiology (ESC). Eur. Heart J., 35(41):2873-2926. [doi:10.1093/eurheartj/ ehu281]

Hayashida, K., Bouvier, E., Lefèvre, T., et al., 2013. Transcatheter aortic valve implantation for patients with severe bicuspid aortic valve stenosis. Circ. Cardiovasc. Interv., 6(3):284-291. [doi:10.1161/CIRCINTERVENTIONS. 112.000084]

Himbert, D., Pontnau, F., Messika-Zeitoun, D., et al., 2012. Feasibility and outcomes of transcatheter aortic valve implantation in high-risk patients with stenotic bicuspid aortic valves. Am. J. Cardiol., 110(6):877-883. [doi:10. 1016/j.amjcard.2012.04.064]

Kochman, J., Huczek, Z., Scisło, P., et al., 2014. Comparison of one- and 12-month outcomes of transcatheter aortic valve replacement in patients with severely stenotic bicuspid versus tricuspid aortic valves (results from a multicenter registry). Am. J. Cardiol., 114(5):757-762. [doi: 10.1016/j.amjcard.2014.05.063]

Leon, M.B., Smith, C.R., Mack, M., et al., 2010. Transcatheter aortic-valve implantation for aortic stenosis in patients who cannot undergo surgery. N. Engl. J. Med., 363(17): 1597-1607. [doi:10.1056/NEJMoa1008232]

Leon, M.B., Piazza, N., Nikolsky, E., et al., 2011. Standardized endpoint definitions for transcatheter aortic valve implantation clinical trials: a consensus report from the Valve Academic Research Consortium. J. Am. Coll. Cardiol., 57(3):253-269. [doi:10.1016/j.jacc.2010.12.005]

Nistri, S., Basso, C., Marzari, C., et al., 2005. Frequency of bicuspid aortic valve in young male conscripts by echocardiogram. Am. J. Cardiol., 96(5):718-721. [doi:10.1016/j. amjcard.2005.04.051]

Roberts, W.C., 1970. The congenitally bicuspid aortic valve: a study of 85 autopsy cases. Am. J. Cardiol., 26(1):72-83. [doi:10.1016/0002-9149(70)90761-7]
Roberts, W.C., Ko, J.M., 2005. Frequency by decades of unicuspid, bicuspid, and tricuspid aortic valves in adults having isolated aortic valve replacement for aortic stenosis, with or without associated aortic regurgitation. Circulation, 111(7):920-925. [doi:10.1161/01.CIR.0000 155623.48408.C5]

Sievers, H.H., Schmidtke, C., 2007. A classification system for the bicuspid aortic valve from 304 surgical specimens. J. Thorac. Cardiovasc. Surg., 133(5):1226-1233. [doi:10. 1016/j.jtcvs.2007.01.039]

Smith, C.R., Leon, M.B., Mack, M.J., et al., 2011. Transcatheter versus surgical aortic-valve replacement in high-risk patients. N. Engl. J. Med., 364(23):2187-2198. [doi:10. 1056/NEJMoa1103510]

Tutar, E., Ekici, F., Atalay, S., et al., 2005. The prevalence of bicuspid aortic valve in newborns by echocardiographic screening. Am. Heart J., 150(3):513-515. [doi:10.1016/j. ahj.2004.10.036]

Wijesinghe, N., Ye, J., Rodés-Cabau, J., et al., 2010. Transcatheter aortic valve implantation in patients with bicuspid aortic valve stenosis. JACC Cardiovasc. Interv., 3(11):1122-1125. [doi:10.1016/j.jcin.2010.08.016]

Zegdi, R., Ciobotaru, V., Noghin, M., et al., 2008. Is it reasonable to treat all calcified stenotic aortic valves with a valved stent? Results from a human anatomic study in adults. J. Am. Coll. Cardiol., 51(5):579-584. [doi:10. 1016/j.jacc.2007.10.023]

Zegdi, R., Lecuyer, L., Achouh, P., et al., 2010. Increased radial force improves stent deployment in tricuspid but not in bicuspid stenotic native aortic valves. Ann. Thorac. Surg., 89(3):768-772. [doi:10.1016/j.athoracsur.2009.12. 022]

\section{中文概要}

题 目: 经导管主动脉瓣置入术在中国人群二叶式主动脉 瓣重度狭窄中的安全性和有效性评估

目 的: 评估经导管主动脉瓣置入术在中国人群二叶式主 动脉瓣重度狭窄中的安全性和有效性。

创新点: 首次在中国人群比较经导管主动脉瓣置入术在二 叶式和三叶式主动脉瓣重度狭窄中的安全性和 有效性。

方 法: 选取 2013 年 3 月至 2014 年 9 月行经导管主动脉 瓣置入术的 40 位主动脉瓣狭窄的患者, 分二叶式 和三叶式主动脉瓣两组, 比较基线水平、手术以 及随访 1 月结果的差别。

结 论: 经导管主动脉瓣置入术在中国人群中二叶式主动 脉瓣重度狭窄中的应用是安全有效的。

关键词: 主动脉瓣狭窄; 二叶式主动脉瓣; 经导管主动脉 瓣置入术 